\title{
Gender and Iron Genes May Modify Associations Between Brain Iron and Memory in Healthy Aging
}

\author{
George Bartzokis*,1,2,3, Po H Lư ${ }^{4}$, Kathleen Tingus ${ }^{4}$, Douglas G Peters ${ }^{1,3}$, Chetan P Amar ${ }^{1,3}$, \\ Todd A Tishler ${ }^{1,3}$, J Paul Finn ${ }^{5}$, Pablo Villablanca ${ }^{5}$, Lori L Altshuler', Jim Mintz ${ }^{6}$, Elizabeth Neely ${ }^{7}$ and \\ James R Connor
}

'Department of Psychiatry and Biobehavioral Sciences, The David Geffen School of Medicine at UCLA, Los Angeles, CA, USA; '2Laboratory of Neuroimaging, Department of Neurology, Division of Brain Mapping, UCLA, Los Angeles, CA, USA; ${ }^{3}$ Department of Psychiatry, Greater Los Angeles VA Healthcare System, Los Angeles, CA, USA; ${ }^{4}$ Department of Neurology, The David Geffen School of Medicine at UCLA, Los Angeles, CA, USA; ${ }^{5}$ Department of Radiology, The David Geffen School of Medicine at UCLA, Los Angeles, CA, USA; ${ }^{6}$ Department of Epidemiology and Biostatistics, University of Texas Health Science Center at San Antonio, San Antonio, TX, USA; ${ }^{7}$ Department of Neurosurgery, Penn State Hershey Medical Center, Hershey, PA, USA

\begin{abstract}
Brain iron increases with age and is abnormally elevated early in the disease process in several neurodegenerative disorders that impact memory including Alzheimer's disease (AD). Higher brain iron levels are associated with male gender and presence of highly prevalent allelic variants in genes encoding for iron metabolism proteins (hemochromatosis H63D (HFE H63D) and transferrin C2 (TfC2)). In this study, we examined whether in healthy older individuals memory performance is associated with increased brain iron, and whether gender and gene variant carrier (IRON +) vs noncarrier (IRON-) status (for HFE H63D/TfC2) modify the associations. Tissue iron deposited in ferritin molecules can be measured in vivo with magnetic resonance imaging utilizing the field-dependent relaxation rate increase (FDRI) method. FDRI was assessed in hippocampus, basal ganglia, and white matter, and IRON+ vs IRON- status was determined in a cohort of 63 healthy older individuals. Three cognitive domains were assessed: verbal memory (delayed recall), working memory/attention, and processing speed. Independent of gene status, worse verbal-memory performance was associated with higher hippocampal iron in men $(r=-0.50, p=0.003)$ but not in women. Independent of gender, worse verbal working memory performance was associated with higher basal ganglia iron in IRON - group $(r=-0.49, p=0.005)$ but not in the IRON + group. Between-group interactions $(p=0.006)$ were noted for both of these associations. No significant associations with white matter or processing speed were observed. The results suggest that in specific subgroups of healthy older individuals, higher accumulations of iron in vulnerable gray matter regions may adversely impact memory functions and could represent a risk factor for accelerated cognitive decline. Combining genetic and MRI biomarkers may provide opportunities to design primary prevention clinical trials that target high-risk groups.

Neuropsychopharmacology (201 I) 36, 1375-1384; doi:10.1038/npp.2011.22; published online 9 March 2011
\end{abstract}

Keywords: memory; iron; gene; sex; dementia; treatment

\section{INTRODUCTION}

Iron is essential for cell function, however, elevated tissue iron can promote tissue-oxidative damage to which the brain is especially vulnerable (Halliwell and Gutteridge, 1985; Kell, 2009; Zecca et al, 2004). Abnormally high brain iron levels are observed in age-related degenerative diseases such as Alzheimer's disease (AD), dementia with Lewy

*Correspondence: Dr G Bartzokis, Department of Psychiatry and Biobehavioral Sciences, The David Geffen School of Medicine at UCLA, 300 UCLA Medical Plaza, Suite 2200, Los Angeles, CA 900956968, USA, Tel: + I 310206 3207, Fax: + I 310268 3266,

E-mail: gbar@ucla.edu

Received 30 September 2010; revised 21 January 201 I; accepted 22 January 2011 bodies (DLB), and Parkinson's disease (PD) (Bartzokis et al, 2007a; Kell, 2009). Brain iron levels increase with age (Bartzokis et al, 2007c; Hallgren and Sourander, 1958) and recent studies reveal elevated levels even in preclinical stages of $\mathrm{AD}$ (Lavados et al, 2008; Smith et al, 2010), suggesting an accelerated trajectory of brain iron accumulation may be occurring during the transition from healthy aging into preclinical stages and eventually dementia (Bartzokis, 2009).

Age-related dementing disorders such as $\mathrm{AD}$ are characterized by progressive memory deficits that begin developing years before the diagnosis can be made (Amieva et al, 2008; den Heijer et al, 2006). The hippocampus (Hip) is a key region in memory function that is severely affected in aging and dementing disorders such as $\mathrm{AD}$ (Braak and 
Braak, 1991; Squire and Zola-Morgan, 1991). Hippocampal iron levels increase with age in healthy individuals (Bartzokis et al, 2007a) and postmortem studies have shown that hippocampal iron is increased in $\mathrm{AD}$ beyond levels of non-demented controls (Bouras et al, 1997; Deibel et al, 1996; Pankhurst et al, 2008; Smith et al, 1997).

Magnetic resonance imaging (MRI) can be used to indirectly assess relationships between cognition and brain iron in living individuals. Several methods of varying sensitivity and specificity have been published (reviewed in Haacke et al, 2005; Pfefferbaum et al, 2009). MRI can measure brain iron levels through the effect of iron on transverse relaxation rates $\left(R_{2}\right)$ (Bartzokis et al, 1993; Bartzokis et al, 1994; Vymazal et al., 1996a; Yao et al, 2009). The bulk of brain iron is stored in ferritin molecules (Floyd and Carney, 1993; Morris et al, 1992) and an in vivo MRI method called field-dependent relaxation rate $\left(R_{2}\right)$ increase (FDRI) can measure their iron content (Bartzokis et al, 1993; Bartzokis et al, 1994). Briefly, FDRI is the difference in measures of brain $R_{2}$ obtained with two different fieldstrength MRI instruments. The FDRI is specifically associated with the total iron contained in ferritin molecules (Bartzokis et al, 1993; Vymazal et al, 1996a) and has been shown to be independent of the amount of iron loading (number of iron atoms per molecule of ferritin) (Vymazal et al, 1996b) and to increase linearly with field-strength (Bartzokis et al, 1993; Gossuin et al, 2004; Vymazal et al, 1996a; Vymazal et al, 1995a; Vymazal et al, 1996b; Yao et al, 2009). In vivo FDRI data has been validated by demonstrating very high correlations with published postmortem data on adult human brain iron distribution as well as replicating the striking age-related increases in iron levels in basal ganglia regions documented in postmortem studies (Bartzokis et al, 2007c; Hallgren and Sourander, 1958; Klintworth, 1973). Thus, FDRI measures the iron contained in ferric oxyhydroxide particles that form the mineral core of ferritin molecules. In human tissue, ferritin and its breakdown product (hemosiderin) are the only known physiologic sources of such particles (Bartzokis et al, 1993; Bulte et al, 1997; Vymazal et al, 1996a; Vymazal et al, 1995b). The FDRI measure will therefore be referred herein as ferritin iron (Bartzokis et al, 1999; Bartzokis et al, 2000).

Recently, Ding et al (2009) used a phase-shift imaging MRI technique that, amongst other things, is affected by tissue iron, and reported that increased iron in the Hip of subjects with $\mathrm{AD}$ may be related to worse cognitive performance and duration of illness. Whether age-related increases in hippocampal iron levels in healthy individuals may represent a trajectory of increasing risk of cognitive decline into AD (Bartzokis, 2009) and are associated with decreased memory performance have yet to be assessed. Herein we present the first study that examines associations between ferritin iron levels in the Hip as well as basal ganglia and white matter regions on memory and processing speed of healthy older individuals.

$\mathrm{We}$, recently, observed higher ferritin iron in men than women (Bartzokis et al, 2007c) and suggested that the increased levels may contribute to the risk of developing neurodegenerative diseases at earlier ages in men (Bartzokis et al, 2007a; Bartzokis et al, 2004; Raber et al, 2004). We also observed that gene variants involved in iron metabolism (hemochromatosis H63D (HFE H63D) and transferrin C2
(TfC2) variants) are associated with higher brain iron levels in healthy older men (Bartzokis et al, 2010). These two gene variants are highly prevalent (affecting approximately 50\% of the population), and some studies have shown an association of these variants with higher risk of developing $\mathrm{AD}$ (Connor and Lee, 2006; Lehmann et al, 2010; Sampietro et al, 2001; Moalem et al, 2000). We therefore examined memory function in the context of gender and the presence $($ IRON + ) or absence (IRON-) of these iron gene variants.

We hypothesized that even in healthy individuals, agerelated increases in ferritin iron levels in the Hip, which is damaged early and severely in dementia-causing diseases such as AD and DLB (Braak et al, 1996; Kotzbauer et al, 2001), will negatively impact memory function (Bartzokis et al, 2007a; Bartzokis et al, 2007c; Bartzokis et al, 2004). Based on data that men may develop neurodegenerative diseases at younger ages (Barker et al, 2002; Friedman, 1994; Miech et al, 2002; Pantelatos and Fornadi, 1993; Raber et al, 2004) (reviewed in Bartzokis et al, 2004), we also hypothesized that healthy older men may be at increased risk for such iron-associated declines in memory function compared to women (Bartzokis et al, 2007c; Bartzokis et al, 2004). In exploratory analyses we also examined the effects of gender and iron gene variants (HFE H63D and TfC2) in basal ganglia and white matter regions on working memory/ attention and processing speed functions.

\section{SUBJECTS AND METHODS}

The subjects, imaging, and genetic methods were described in detail in prior publications (Bartzokis et al, 2010; Bartzokis et al, 2007c) and will only be summarized here.

\section{Subjects}

Normal older adult volunteers were recruited from the community and hospital staff for a study of healthy aging (Bartzokis et al, 2007c). Potential subjects were excluded if they had a history of neurological disorder or a family history of $\mathrm{AD}$ or other neurodegenerative disorder, psychiatric illness (including drug or alcohol abuse), or head injury resulting in loss of consciousness for more than $10 \mathrm{~min}$. The subjects were physically very healthy and were excluded if they were obese, or if they had a current or prior serious illness, or a medical history of diabetes, cardiovascular disease, or difficult-to-control hypertension. They were independently functioning and had no evidence of neurocognitive impairment or gross neurological abnormalities on clinical interview and brief neurological examination with the study PI. The final population of 63 individuals contained 33 men and 30 postmenopausal women ranging in age from 55 to 76 years (mean $=67.0$ years, $\mathrm{SD}=6.1)$. The sample averaged 15.8 years of education $(\mathrm{SD}=2.5$; range $=10-23$ years $)$ and their reported racial composition was 45 (71\%) Caucasian, 13 (21\%) Asian, and 5 (8\%) African-American. All subjects received written and oral information about the study and signed written informed consents approved by the local Institutional Review Board prior to study participation.

The participants were first assessed and evaluated with MRI, clinical assessment, and neurocognitive measures. The 
subjects were later genotyped for the presence of $\mathrm{TfC} 2$ or HFE H63D and C282Y genes. There were no carriers of the rare $\mathrm{HFE} \mathrm{C} 282 \mathrm{Y}$ variant in this sample so the only genes present were TfC2 and HFE H63D (Bartzokis et al, 2010). Of the total sample, 32 subjects had one or both genes (IRON + group; 18 males, 14 females) while 31 had neither gene (IRON- group; 15 males, 16 females).

\section{Neurocognitive Measures}

The neurocognitive measures were collected within one month of the MRI scan.

Memory related. The California Verbal Learning Test (CVLT; Delis et al, 1987) is a measure of rote verbal learning and memory in which a list of 16 words is presented over 5 trials and recalled after an interference list and again after a 20-min delay. The total number of words recalled after 20 min serves as a measure of delayed memory for unstructured verbal material.

Verbal working memory/attention related. Auditory Consonant Trigrams (ACT; Peterson and Peterson, 1959) is a sensitive measure of working memory, requiring the subject to sustain information in short-term memory while performing other cognitive operations. Basic and complex attention span was assessed using the Digit Span subtest from the WAIS III (Wechsler, 1997).

Processing speed related. Trailmaking Test_- part A and B (Reitan and Wolfson, 1985) assesses information processing speed, visuomotor tracking, and mental flexibility. Part A requires subjects to rapidly connect consecutively numbered circles, and part B requires subjects to consecutively connect circles containing numbers and letters by alternating between the two sequences (e.g., 1-A-2-B). Time to complete the task serves as the variable of interest. Digit Symbol subtest from the WAIS-R (Wechsler, 1981) involves rapid copying of symbols and integrates several cognitive processes including psychomotor speed, visual scanning, and simple constructional ability. The score reflects number of symbols copied within 90 seconds.

\section{MRI Protocol}

The methods have been previously described in detail (Bartzokis et al, 1993; Bartzokis et al, 1994; Bartzokis et al, 2007c). The participants were scanned using two MRI instruments ( 1.5 and $0.5 \mathrm{~T}$ ) within $1 \mathrm{~h}$ of each other using the same imaging protocol. Coronal and sagittal pilot scans were first obtained to specify the location and spatial orientation of the head and the position of the axial image-acquisition grid. The axial image-acquisition sequence acquired interleaved contiguous slices using a Carr-Purcell-Meiboom-Gill dual spin-echo sequence (time to repetition $(\mathrm{TR})=2500 \mathrm{~ms}$; time to echo $(\mathrm{TE})=20$ and $90 \mathrm{~ms} ; 2$ excitations; $3 \mathrm{~mm}$ slice thickness; 192 gradient steps; and $25 \mathrm{~cm}$ field-of-view). The coronal and sagittal pilot scans were used to determine the alignment and accuracy of head repositioning in the second MRI instrument (Bartzokis et al, 1993; Bartzokis et al, 1994).

Data for each region of interest (ROI; depicted in Figure 1) were obtained from contiguous pairs of slices. The slice containing the anterior commissure and the slice immediately superior to it were used to obtain the putamen $(\mathrm{P})$ and globus pallidus $(G)$ transverse relaxation time $\left(T_{2}\right)$ data. The third and fourth slices above the anterior commissure were used to obtain the $T_{2}$ data for caudate nucleus (C) and the second and third slices superior to the orbitofrontal gray matter were used to obtain the frontal lobe white matter (Fwm) data. For the genu of the corpus callosum (Gwm), the two slices on which the angle formed by the left and the right sides of the genu appeared the most linear were chosen in order to obtain a sample that would be consistently in the middle of the structure, which contains primarily fibers connecting the prefrontal cortices. For the splenium of the corpus callosum (Swm), the second and third lowest slices on which the fibers of the splenium connected in the midline were chosen in order to sample primarily the lower half of the splenium that contains predominantly primary sensory (visual) fibers. For thalamus (T), the second and third highest slices on which thalamus appears are chosen. The lateral border of the ROI is drawn along the white matter of the internal capsule using the $\mathrm{TE}=20$ image. The medial and inferior portions bordering CSF are defined using the TE $=90$ image. For Hip, the two contiguous slices
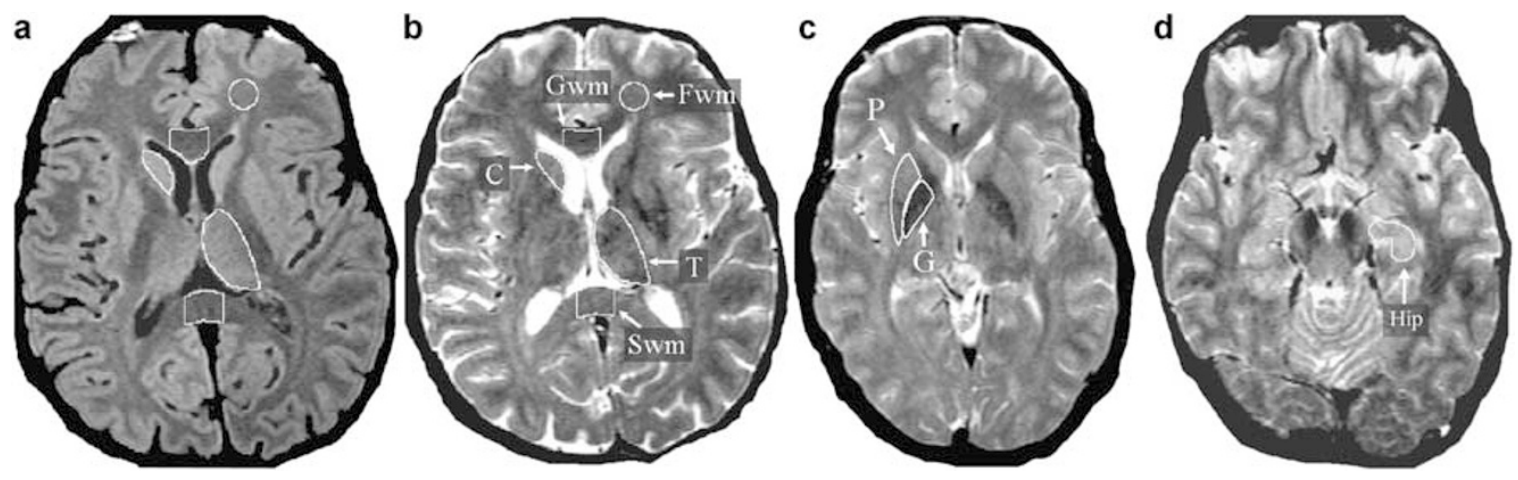

Figure I Regions of interest (ROIs). ROI definition is depicted on axial MRI TE20 (a) and TE90 (b-d). The TE20 has optimal contrast between gray (appears light gray) and white matter (appears dark gray). The TE90 has optimal contrast between brain (appears gray) and CSF (appears white). Both TE20 and TE90 images are used to draw each ROI as this combination of images maximizes contrast needed for accurate ROI definition. As an example, the use of both contrasts is depicted in the thalamus ROI that borders CSF medially and white matter laterally and posteriorly (a and b). Data for each ROI are obtained from contiguous pairs of slices. Only one hemisphere ROI is depicted on the figures although ROls are measured bilaterally for all regions except for midline corpus callosum regions (Gwm and Swm). Please see text ('MRI Protocol') for further details. 
that contained the largest areas of these structures were used in the data analysis. The Hip measure was obtained from the anterior third of the structure and was limited by drawing a horizontal line at the level of the cerebral peduncle to exclude any tissue posterior to that line (Bartzokis et al, 2010; Bartzokis et al, 2007c).

\section{Image Analysis}

Transverse relaxation times $\left(T_{2}\right)$ were calculated for each voxel by an automated algorithm from the two signal intensities ( $\mathrm{TE}=20$ and 90) of the dual spin-echo sequence to produce gray-scale encoded $T_{2}$ maps of the brain (Bartzokis et al, 1994). The $T_{2}$ measures were extracted using an Apple Macintosh-configured image analysis workstation. $T_{2}$ data for each of the ROIs were obtained from contiguous pairs of slices. The $R_{2}$ was calculated as the reciprocal of $T_{2} \times 1000 \mathrm{~ms} / \mathrm{s}$. The average $R_{2}$ of the two slices from both hemispheres were the final measures used in the subsequent analyses. The FDRI measure was calculated as the difference in $R_{2}$ (high-field $R_{2}$-low-field $\mathrm{R}_{2}$ ). Test-retest reliability for FDRI measures was very high with intraclass correlation coefficients ranging from 0.88 to $0.99(p<0.0023)$ (Bartzokis et al, 1993; Bartzokis et al, 1994).

\section{Data Analyses}

Parametric approaches were used to assess the impact of the iron gene variants and gender on the association between brain iron and cognition. We first performed multiple regression analysis that crosses cognitive scores with measures of brain iron. To supplement the regression analyses, Pearson's correlations were conducted to examine the relationship between brain iron measures and cognitive performance in relevant subgroups (eg, stratified by gender and gene grouping). Differences between correlations were then tested by the non-parametric approach of normal curve test on Fisher's $z$-transformed values. As in this restricted age range the FDRI measure was not significantly associated with age in any of the regions, age was not included in the analyses.

\section{Hippocampal Iron and Episodic Memory}

A specific hypothesis regarding hippocampal iron and episodic memory performance was tested by performing a multiple regression analysis that crosses the CVLT delayed recall score with the FDRI measure of iron in the Hip. The memory measure was the dependent variable, and the independent variables were gender, iron genes (presence or absence of genes), and the Hip FDRI measure. The independent variable factors were fully crossed and our interest focused on the two-factor interactions of gene $\times$ brain iron measure and the three-factor gene $\times$ brain iron $\times$ gender interactions.

\section{Basal Ganglia and White Matter Iron and Non-Memory Cognitive Performance}

We then assessed whether the iron genes impact the association between iron levels in other brain regions and non-memory aspects of cognitive functioning. For the purpose of data reduction, principal components analysis was performed separately for the cognitive tasks and the measures of brain iron. In each case, the analysis suggested retention of two components for rotation, and the interpretation was based on eigenvalues $>1$ and a clear break in the scree curve. Substantive interpretations of the components were based on highest loadings for each variable. In the cognitive variables, the components were interpreted as being related to processing speed (Trailmaking Test - parts A and B, Digit Symbol) and auditory working memory/ attention (ACT, Digit Span). In the FDRI measures of brain iron, the components represented measures of the basal ganglia (caudate, globus pallidus, and putamen) and white matter (frontal, splenium of the corpus callosum, genu of the corpus callosum, and thalamus). Component scores were computer generated by regression-based scoring, producing standardized scores with mean $=0$ and standard deviation $=1$.

Using the same statistical methodologies detailed above, four multiple regression analyses were generated by crossing the two cognitive factors (working memory/attention and processing speed) with the two measures of brain iron (basal ganglia and white matter). The two cognitive measures were the dependent variables and the independent variables were gender, iron genes (presence $($ IRON + ) or absence (IRON-) of genes), and either the basal ganglia or white matter component score. The independent variable factors were fully crossed, and our interest focused on the two-factor interactions of gene $\times$ brain iron measure and the three-factor interactions of gene $\times$ brain iron $\times$ gender.

All $p$-values were assessed as significant at an $\alpha$ level of 0.05 . The $p$-values for the multiple regression and Pearson's correlation analyses testing the associations between basal ganglia and white matter iron with nonmemory cognitive performance were assessed as significant at an $\alpha$ level of 0.0125 to reflect Bonferroni correction for the four comparisons.

\section{RESULTS}

The iron absorption genes did not have a significant effect on the relationship between Hip iron and CVLT. The analysis of Hip iron and CVLT performance yielded a significant interaction of gender by iron level $(F=10.56$, $\mathrm{df}=1,55, p=0.002)$. Pearson's correlation analyses indicated a significant negative association between the Hip iron and CVLT in men $(N=33, r=-0.50, \mathrm{df}=31$, $p=0.003)$, and a nonsignificant positive association between these two measures in women $(N=30, r=0.19$, $\mathrm{df}=28, p=0.31$ ). The difference between these correlation coefficients was significant and similar to the result from the more complex regression model above (Fisher's $z$-test: $t=2.74, p=0.006)$. The results are depicted in Figure 2 and were not substantially altered after controlling for age in the analyses.

The regression models based on iron and cognitive components yielded a significant effect of the iron genes on the association between basal ganglia iron level and the working memory/attention score (interaction $\mathrm{F}=8.15$, $\mathrm{df}=1,55, p=0.006)$. As gender was not a significant factor 


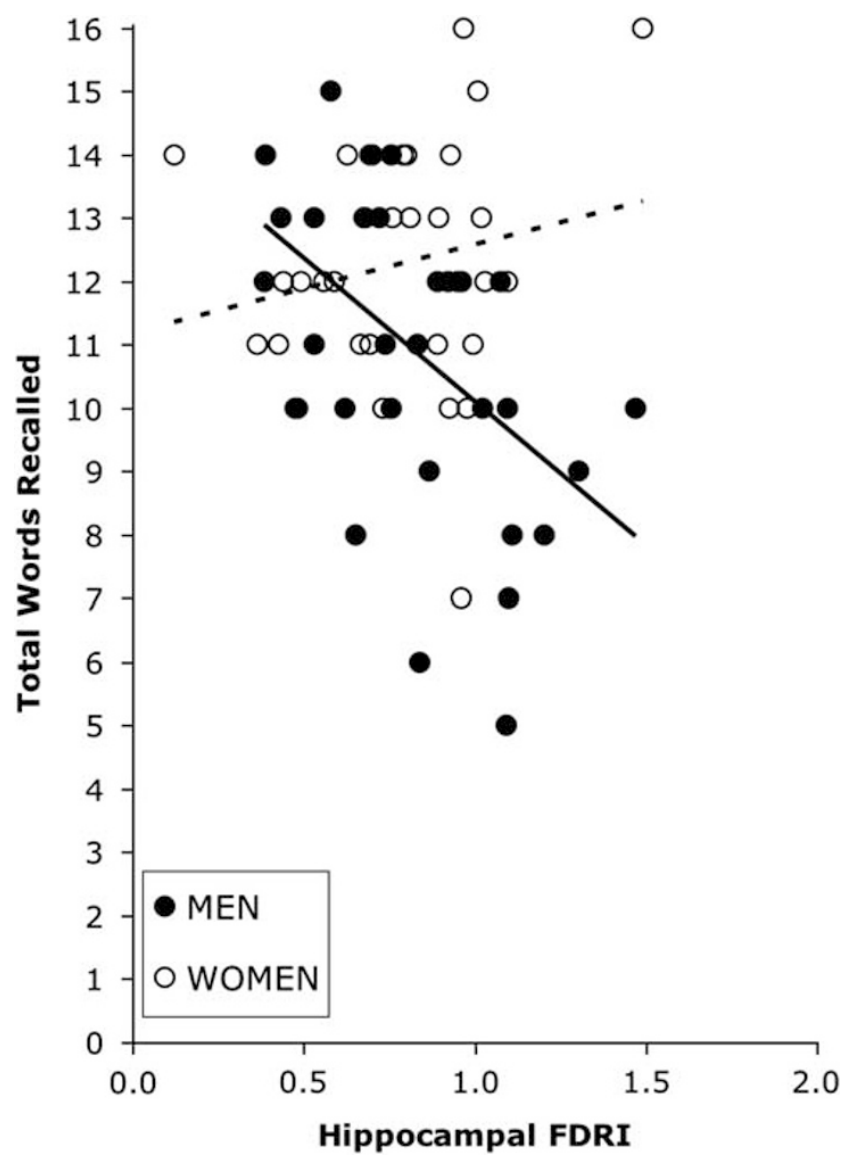

Figure 2 Memory function vs hippocampal ferritin iron in healthy older men and women. Men: $r=-0.50, p=0.003$. Women: $r=0.19, p=0.31$. Memory function: assessed using The California Verbal Learning Test (CVLT) (Delis et al, 1987), which is a measure of rote verbal learning and memory (number of words recalled after a 20-min delay). FDRI, fielddependent transverse relaxation rate $\left(R_{2}\right)$ increase, an MRI measure of ferritin iron (the iron content of ferritin molecules).

in this model, it was not examined separately. We computed the Pearson's correlation between basal ganglia iron and working memory/attention and found a significant negative relationship in the IRON- group $(N=31, r=-0.49$, $\mathrm{df}=29, p=0.005)$; thus for those without either of the iron genes (H63D and TfC2), increased basal ganglia iron was significantly associated with worse performance in working memory/attention. A positive but nonsignificant association was observed in the IRON + group $(N=32, r=0.19$, $\mathrm{df}=30, p=0.30)$. The difference in correlation coefficients between the IRON + and IRON- groups were statistically significant and mirrored the regression result (Fisher's $z$-test: $t=2.75, p=0.006)$. The results are depicted in Figure 3 and were not substantially altered by controlling for age in the analyses. The results from the multiple regression and Pearson's correlation analyses remain significant after Bonferroni correction $(\times 4$ tests $)$ for multiple comparisons (corrected: $p=0.024$ and $p=0.020$, respectively). None of the analyses involving white matter iron approached significance.

When the above regression analyses were confined to only Caucasian subjects $(N=45)$, all the findings remained essentially unchanged.

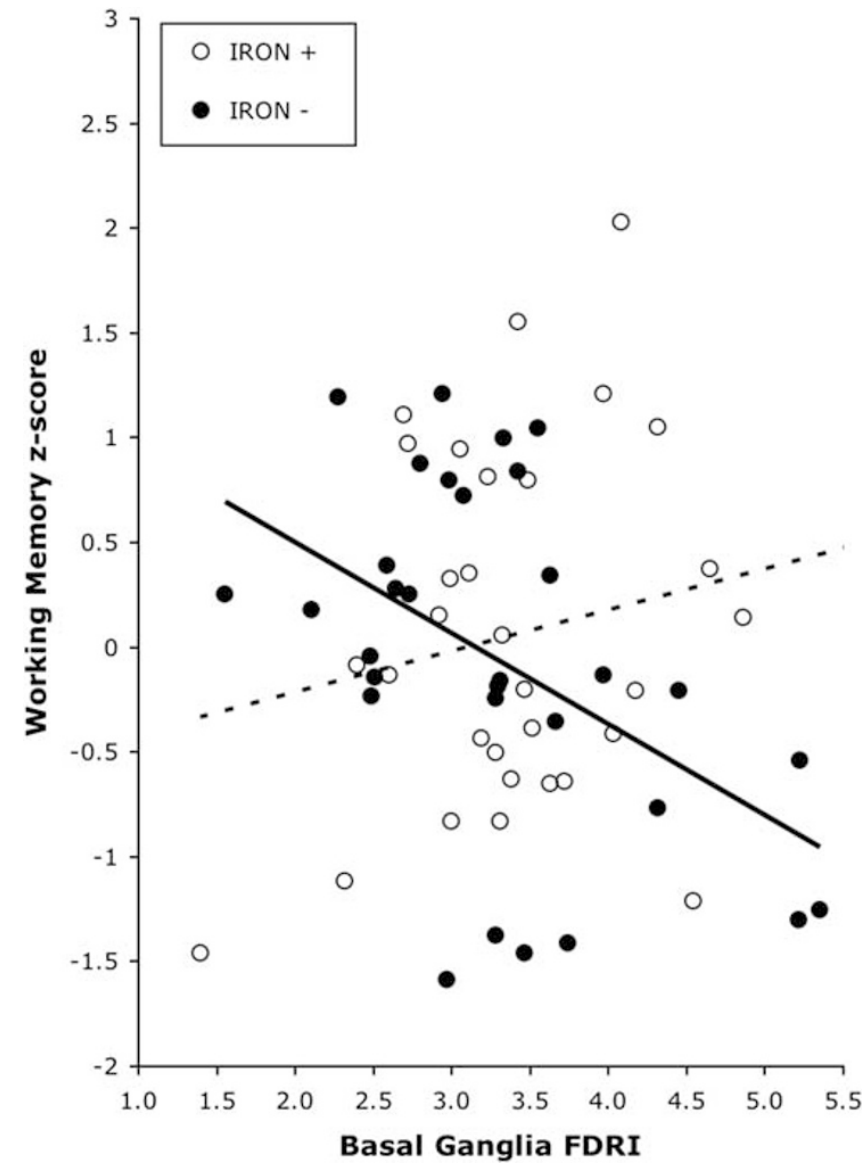

Figure 3 Working memory function vs basal ganglia ferritin iron in healthy older men and women with and without iron genes. IRON-: wild type gene carriers $(r=-0.49, p=0.005)$. IRON +: carriers of either transferrin C2 (TfC2) and/or hemochromatosis H63D (HFE H63D) gene variants $(r=0.19, p=0.30)$. Working memory function: assessed using a composite score of Auditory Consonant Trigrams (ACT; Peterson and Peterson, 1959) and Digit Span, which require subjects to either manipulate information or simultaneously perform another cognitive operation while sustaining the original information in short-term memory. Basal ganglia: composite score of caudate, putamen, and globus pallidus. FDRI, fielddependent transverse relaxation rate $\left(R_{2}\right)$ increase, an MRI measure of ferritin iron (the iron content of ferritin molecules).

\section{DISCUSSION}

This is the first demonstration that in healthy older men, declarative memory function may be adversely affected by increased Hip ferritin iron. A male-specific association is in line with our previously observed higher brain iron levels in men compared to women across the lifespan (Bartzokis et $a l, 2007 \mathrm{c}$ ) and increased iron levels in male carriers of highly prevalent allelic variants of genes (HFE H63D and/or TfC2) that encode proteins involved in iron metabolism (Bartzokis et al, 2010). These observations are consistent with the proposition that a portion of the suggested earlier age at onset in men of neurodegenerative diseases such as AD, PD, and DLB (Barker et al, 2002; Raber et al, 2004) may be accounted for, at least in part, by their higher brain ferritin iron (Bartzokis et al, 2004) (reviewed in Bartzokis et al, 2007c). In women, the iron-memory association was significantly different from the one observed in men. The 
association could be substantially modified in women because of the gender-specific pattern of iron requirements such as those produced by menstruation and/or metabolism (see below) (Coppus et al, 2009; Whitfield et al, 2003) (reviewed in Bartzokis et al, 2007c).

Animal data suggest that during early postnatal development, increases in Hip ferritin iron are necessary for normal cognitive function and memory (Carlson et al, 2009; Georgieff, 2008; Schmidt et al, 2007; Shoham and Youdim, 2002; Siddappa et al, 2004) and that early deficits can have long-lasting detrimental effects on cognition even when corrected through supplementation (Schmidt et al, 2007). Differentiating oligodendrocytes have very high iron requirements and may have a critical role in brain iron uptake (reviewed in Bartzokis et al, 2007a; Bartzokis et al, 2007b). This may be especially important in the hippocampus where $50 \%$ of oligodendrocytes are juxtaposed directly on blood vessels and may thus be in a position to acquire iron directly from the vasculature (Vinet et al, 2010). On the other hand, iron overload may also cause cognitive deficits (Maaroufi et al, 2009) and increased neonatal iron intake as well as overexpression of ferritin may be associated with increased risk of neurodegenerative disease in late life (Carlson et al, 2008; Kaur et al, 2006a; Kaur et al, 2006b). It is thus becoming evident that regulating the processes underlying brain iron accumulation trajectories has important consequences throughout the lifespan. Furthermore, many neurodegenerative diseases are associated with dysregulated iron metabolism (Bartzokis, 2009; Kell, 2009; Roth et al, 2010; Zecca et al, 2004). Such dysregulations are often manifested as increases in tissue iron that go beyond those observed in healthy individuals (reviewed in Bartzokis et al, 2007c; Kell, 2009; Zecca et al, 2004). One mechanism leading to such iron increases could result from abnormal intracellular and extracellular protein deposits (proteinopathies) triggering inappropriate iron accumulation in multiple disorders (Singh et al, 2009; Smith et al, 1997) (reviewed in Bartzokis, 2009; Bartzokis et al, 2007a).

Iron regulation in neurons seems to be strongly dependent on the ferroxidase activity of amyloid precursor protein (APP) that is responsible for removing excess iron (Duce et al, 2010) and may thus mitigate intracellular iron-catalyzed oxidative damage (Halliwell and Gutteridge, 1988). The iron-management function of APP is also supported by the observation that APP transcription is controlled, at least in part, by the same iron-sensing mechanism that controls transcription of two canonical iron metabolism proteins: ferritin and transferrin (Cahill et al, 2008). Neurons may be especially susceptible to excess iron because of the disproportionately long processes (axons) connecting neuron body to synapses, and APP is well suited for its iron management function because of its presence throughout the neuron. The other well-known function of APP is to adhere the 'cargo' (eg, vesicles) to the 'motors' powering the fast axonal transport (FAT) that moves supplies from the neuron body to axons and synapses, and back. The two-way FAT traffic thus automatically provides the ferroxidase function of APP throughout, and especially near mitochondria that amass all along the axon at the nodes of Ranvier as well as at distant synapses. Removal of iron liberated from any source, such as damaged iron-rich mitochondria being transported back to the neuronal body for degradation, would be crucial throughout the FAT transport system (reviewed in Bartzokis, 2009).

The Hip region has been shown to have elevated iron levels in AD (Deibel et al, 1996; Good et al, 1992; Smith et al, 1997; Thompson et al, 1988) (Bartzokis et al, unpublished data), and is affected early and severely in age-related proteinopathies such as AD and DLB (Braak et al, 1996; Kotzbauer et al, 2001) that cause the vast majority (over 70\%) of dementia (Barker et al, 2002; Fratiglioni et al, 2000; Lobo et al, 2000). The observation that increased hippocampal iron is associated with poorer memory function even in healthy older individuals supports the suggestion that the 'normal' trajectory of age-related increases in brain ferritin iron may represent an underlying risk factor for age-related degenerative brain diseases (Chen et al, 2009) (reviewed in Bartzokis, 2009). Age-related accumulations of brain iron in structures such as basal ganglia and Hip of healthy individuals (Bartzokis et al, 2007c; Hallgren and Sourander, 1958) may be conceptualized as 'normal' trajectories toward brain iron overload of vulnerable regions (Bartzokis, 2009) that may be modified by gender and genetic differences (Bartzokis et al, 2010; Bartzokis et al, 2007c). These differences may modify trajectories of iron accumulation from normal age-related memory declines (Figures 2 and 3) into preclinical stages of degeneration and eventually dementias (Bartzokis, 2009; Lavados et al, 2008; Smith et al, 1997; Smith et al, 2010).

As reviewed above, however, both iron deficiency and brain iron excess can have deleterious consequences on cognition. In old age, adequate iron levels are essential for the continual process of myelin repair/replacement, a key process in maintaining cognitive functions (reviewed in Bartzokis, 2009). Thus the opposite association we observed in men compared to that in women in the Hip-memory correlations are not entirely unexpected given known gender differences in iron status. Women begin their postmenopausal years in a state of relative peripheral iron deficiency compared with men and their iron levels increase for the first 15-20 years after menopause without fully 'catching up' to those in men (Whitfield et al, 2003). As the peripheral iron levels can influence brain iron accumulation (Bartzokis et al, 2007c; House et al, 2010; Li et al, 2010), these peripheral effects could manifest in the brain. Thus, as the two genders approach older ages, it is possible that the already higher levels of iron observed in men push them into a toxic range earlier as they enter older ages, whereas women, who start with considerably lower iron levels, may initially experience a cognitive benefit from increasing postmenopausal iron levels.

The basal ganglia accumulate markedly more iron with age than most other brain structures (Bartzokis et al, 2007c; Hallgren and Sourander, 1958) and may thus require additional protection from iron-associated toxicity. Further analyses revealed an unexpected modifying effect of prevalent iron metabolism gene variants on the association between basal ganglia ferritin iron and working memory function (Figure 3 ). This observation is consistent with the direct involvement of the basal ganglia in working memory networks (Battig et al, 1960; Chorover and Gross, 1963) (reviewed in Constantinidis and Procyk, 2004; Simpson 
et al, 2010). Striatofrontal networks interconnect specific areas of the prefrontal cortex to subregions of the basal ganglia, forming loops that are intricately involved in higher cognitive functions including working memory (Alexander et al, 1986; Battig et al, 1960; Chorover and Gross, 1963; Landau et al, 2009; Lewis et al, 2004). In iron gene noncarriers (IRON- group), higher brain iron in the basal ganglia was again significantly associated with worse verbal working memory. A different (opposite, albeit nonstatistically significant) relationship was seen in the gene carrier $($ IRON + ) group. These genes have been associated with brain iron uptake as well as complex interactions between basal ganglia ferritin iron levels, metabolic processes, and cognitive functions (Burdo et al, 2004; Lee et al, 2007; Li et al, 2010; Ma et al, 2008; Mitchell et al, 2009). The presence of these genes seems to mitigate the detrimental effect of basal ganglia iron accumulation on working memory function observed in the IRON- group and this may help explain, at least in part, the very high prevalence of these genes in the population.

Animal models support the suggestion that iron overload is associated with memory decline (Maaroufi et al, 2009), and treatment with iron chelators have been reported to result in improved memory even in healthy aged rodents (de Lima et al, 2007). Human studies have also suggested that chelation treatment may be helpful in degenerative brain diseases (reviewed in Kell, 2009). The mechanism of iron toxicity is likely related to promotion of damaging free-radical reactions and associated inflammation (Smith et al, 1997) (reviewed in Kell, 2009). It is thus not surprising that invertebrate data (Drosophila) suggest that age-related iron accumulation is proportional to the rate of aging (Massie et al, 1985) and inhibition of iron absorption prolongs lifespan (Massie et al, 1993). Similarly, human gender differences in longevity have been proposed to relate to reproduction-related iron losses in women (Sullivan, 1989) and life-extending effects of calorie restriction have been associated with reduced dietary iron uptake and lowered iron deposits in tissue (Cook and Yu, 1998; Kastman et al, 2010; Valle et al, 2008; Xu et al, 2008).

Several limitations of the current study also need to be considered. First, strict criteria for inclusion of healthy older individuals and small sample size may underestimate the strength of correlations between tissue iron and cognition by restricting sample variance. Second, interpretation of 'changes' from cross-sectional data must be made with caution (Kraemer et al, 2000), and prospective studies and larger samples are needed to further define age-related trajectories in each gender and genetic subgroup. Third, the absence of a sample of younger adults limits the ability to detect quadratic or other nonlinear associations. Fourth, peripheral iron measures and detailed information on blood loss during life, and other environmental influences such as iron supplementation that may affect brain iron were not available. Fifth, although we observed a significant effect of HFE and TfC2 genes on brain iron levels (Bartzokis et al, 2010) and the basal ganglia-working memory association, these genes likely account for a minority of the variance, much of the genetic influence on iron levels remains unknown (Njajou et al, 2006; Whitfield et al, 2000), and future studies may identify additional genetic influences on brain iron and its impact on cognitive performance. Finally, although reproducible (Bartzokis et al, 1994; Bartzokis et al, 2000) and very highly correlated with postmortem iron levels (Bartzokis et al, 2007c), the FDRI measure specifically quantifies ferritin iron load that may be only indirectly related to the amount of free iron or other transition metals that may be more directly associated with toxicity (Lavados et al, 2008; Rajendran et al, 2009).

The significantly different associations we observed in subgroups suggest that toxic consequences on cognitive function of age-related brain iron increases may differ substantially by gender and genotypes, and that brain iron deposition is a complex multifactorial endophenotype. Treatment efforts to reduce the deleterious effects of brain iron accumulations in old age (Adlard et al, 2008; Cahill et al, 2008; Chen-Roetling et al, 2009; Hider et al, 2008; Mandel et al, 2008; Zecca et al, 2008) (for review see Kell, 2009) should take into consideration that large subgroups of the population may substantially differ in brain iron regulation mechanisms and thus differ in their response to such interventions.

The advent of in vivo neuroimaging methods that can assess tissue ferritin iron deposits on a regional basis with high specificity provides the means to prospectively examine the impact of age-related changes in iron on trajectories of cognitive decline into neurodegenerative diseases. These methods could be used to measure iron accumulations as well as target emerging therapeutic interventions (Adlard et al, 2008; Cahill et al, 2008; ChenRoetling et al, 2009; Hider et al, 2008; Mandel et al, 2008; Zecca et al, 2008) (for review see Kell, 2009) to high-risk groups identified by MRI, genetic, and clinical biomarkers, years before clinical manifestations of disease. Early intervention in higher-risk subgroups may make it possible to increase effectiveness of treatments, decrease the need for more-aggressive approaches at later stages, and may identify heretofore unexplored opportunities for primary prevention for the exponentially increasing burden of age-related neurodegenerative diseases (Bartzokis, 2009; Bartzokis et al, 2007c).

\section{ACKNOWLEDGEMENTS}

This work was supported in part by NIH grants (AG027342 and MH0266029), the Department of Veterans Affairs, the RCS Alzheimer's Foundation, and the George M Leader Family. This work was presented in part at the 451st Annual Winter Conference on Brain Research, Snowbird, Utah, January 2008, and the Alzheimer's Association International Conference on Alzheimer's Disease (ICAD), Vienna, Austria, July 2009.

\section{DISCLOSURE}

The authors declare no conflict of interest.

\section{REFERENCES}

Adlard PA, Cherny RA, Finkelstein DI, Gautier E, Robb E, Cortes $\mathrm{M}$ et al (2008). Rapid restoration of cognition in Alzheimer's transgenic mice with 8-hydroxy quinoline 
analogs is associated with decreased interstitial Abeta. Neuron 59: $43-55$.

Alexander GE, DeLong MR, Strick PL (1986). Parallel organization of functionally segregated circuits linking basal ganglia and cortex. Annu Rev Neurosci 9: 357-381.

Amieva H, Le Goff M, Millet X, Orgogozo JM, Peres K, BarbergerGateau P et al (2008). Prodromal Alzheimer's disease: successive emergence of the clinical symptoms. Ann Neurol 64: 492-498.

Barker WW, Luis CA, Kashuba A, Luis M, Harwood DG, Loewenstein D et al (2002). Relative frequencies of Alzheimer disease, Lewy body, vascular and frontotemporal dementia, and hippocampal sclerosis in the state of Florida brain bank. Alzheimer Dis Assoc Disord 16: 203-212.

Bartzokis G (2009). Alzheimer's disease as homeostatic responses to age-related myelin breakdown. Neurobiol Aging. doi:10.1016/j. neurobiolaging.2009.08.007.

Bartzokis G, Aravagiri M, Oldendorf WH, Mintz J, Marder SR (1993). Field dependent transverse relaxation rate increase may be a specific measure of tissue iron stores. Magn Reson Med 29: 459-464.

Bartzokis G, Cummings JL, Markham CH, Marmarelis PZ, Treciokas LJ, Tishler TA et al (1999). MRI evaluation of brain iron in earlier- and later-onset Parkinson's disease and normal subjects. Magn Reson Imaging 17: 213-222.

Bartzokis G, Lu P, Tishler TA, Peters DG, Kosenko A, Barrall KA et al (2010). Prevalent iron metabolism gene variants associated with increased brain ferritin iron in healthy older men. J Alzheimers Dis 20: 333-341.

Bartzokis G, Lu PH, Mintz J (2007a). Human brain myelination and amyloid beta deposition in Alzheimer's disease. Alzheimers Dement 3: 122-125.

Bartzokis G, Lu PH, Tishler TA, Fong SM, Oluwadara B, Finn JP et al (2007b). Myelin breakdown and iron changes in Huntington's disease: pathogenesis and treatment implications. Neurochem Res 32: 1655-1664.

Bartzokis G, Mintz J, Sultzer D, Marx P, Herzberg JS, Phelan CK et al (1994). In vivo MR evaluation of age-related increases in brain iron. AJNR Am J Neuroradiol 15: 1129-1138.

Bartzokis G, Sultzer D, Cummings BJ, Holt LE, Hance DB, Henderson VW et al (2000). In vivo evaluation of brain iron in Alzheimer's disease and normal controls using magnetic resonance imaging. Arch Gen Psychiatry 57: 47-53.

Bartzokis G, Tishler TA, Lu PH, Villablanca P, Altshuler LL, Carter $\mathrm{M}$ et al $(2007 \mathrm{c})$. Brain ferritin iron may influence age- and gender-related risks of neurodegeneration. Neurobiol Aging 28: 414-423.

Bartzokis G, Tishler TA, Shin I-S, Lu PH, Cummings JL (2004). Brain ferritin iron as a risk factor for age at onset in neurodegenerative diseases. In: LeVine S, Connor J, Schipper $\mathrm{H}$ (eds). Redox-active Metals in Neurological Disorders Ann NY Acad Sci 1012: 224-236.

Battig K, Rosvold HE, Mishkin M (1960). Comparison of the effects of frontal and caudate lesions on delayed response and alternation in monkeys. J Comp Physiol Psychol 53: 400-404.

Bouras C, Giannakopoulos P, Good PF, Hsu A, Hof PR, Perl DP (1997). A laser microprobe mass analysis of brain aluminum and iron in dementia pugilistica: comparison with Alzheimer's disease. Eur Neurol 38: 53-58.

Braak H, Braak E (1991). Neuropathological stageing of Alzheimerrelated changes. Acta Neuropathol (Berl) 82: 239-259.

Braak H, Braak E, Yilmazer D, de Vos RA, Jansen EN, Bohl J (1996). Pattern of brain destruction in Parkinson's and Alzheimer's diseases. J Neural Transm 103: 455-490.

Bulte JW, Miller GF, Vymazal J, Brooks RA, Frank JA (1997). Hepatic hemosiderosis in non-human primates: quantification of liver iron using different field strengths. Magn Reson Med 37: 530-536.
Burdo JR, Simpson IA, Menzies S, Beard J, Connor JR (2004). Regulation of the profile of iron-management proteins in brain microvasculature. J Cereb Blood Flow Metab 24: 67-74.

Cahill CM, Lahiri DK, Huang X, Rogers JT (2008). Amyloid precursor protein and alpha synuclein translation, implications for iron and inflammation in neurodegenerative diseases. Biochim Biophys Acta 1790: 615-628.

Carlson ES, Magid R, Petryk A, Georgieff MK (2008). Iron deficiency alters expression of genes implicated in Alzheimer disease pathogenesis. Brain Res 1237: 75-83.

Carlson ES, Tkac I, Magid R, O'Connor MB, Andrews NC, Schallert $\mathrm{T}$ et al (2009). Iron is essential for neuron development and memory function in mouse hippocampus. J Nutr 139: 672-679.

Chen H, Lukas TJ, Du N, Suyeoka G, Neufeld AH (2009). Dysfunction of the retinal pigment epithelium with age: increased iron decreases phagocytosis and lysosomal activity. Invest Ophthalmol Vis Sci 50: 1895-1902.

Chen-Roetling J, Chen L, Regan RF (2009). Minocycline attenuates iron neurotoxicity in cortical cell cultures. Biochem Biophys Res Commun 386: 322-326.

Chorover SL, Gross CG (1963). Caudate nucleus lesions: behavioral effects in the rat. Science 141: 826-827.

Connor JR, Lee SY (2006). HFE mutations and Alzheimer's disease. J Alzheimers Dis 10: 267-276.

Constantinidis C, Procyk E (2004). The primate working memory networks. Cogn Affect Behav Neurosci 4: 444-465.

Cook CI, Yu BP (1998). Iron accumulation in aging: modulation by dietary restriction. Mech Ageing Dev 102: 1-13.

Coppus AM, Evenhuis HM, Verberne GJ, Visser FE, Eikelenboom $\mathrm{P}$, van Gool WA et al (2010). Early age at menopause is associated with increased risk of dementia and mortality in women with Down syndrome. J Alzheimers Dis 19: 545.

de Lima MN, Dias CP, Torres JP, Dornelles A, Garcia VA, Scalco FS et al (2007). Reversion of age-related recognition memory impairment by iron chelation in rats. Neurobiol Aging 29: 1052-1059.

Deibel MA, Ehmann WD, Markesbery WR (1996). Copper, iron, and zinc imbalances in severely degenerated brain regions in Alzheimer's disease: possible relation to oxidative stress. J Neurol Sci 143: 137-142.

Delis DC, Kramer JH, Kaplan E, Ober BA (1987). California Verbal Learning Test. Psychological Corporation: San Antonio, TX.

den Heijer T, Geerlings MI, Hoebeek FE, Hofman A, Koudstaal PJ, Breteler MM (2006). Use of hippocampal and amygdalar volumes on magnetic resonance imaging to predict dementia in cognitively intact elderly people. Arch Gen Psychiatry 63: 57-62.

Ding B, Chen KM, Ling HW, Sun F, Li X, Wan $\mathrm{T}$ et al (2009). Correlation of iron in the hippocampus with MMSE in patients with Alzheimer's disease. J Magn Reson Imaging 29: 793-798.

Duce JA, Tsatsanis A, Cater MA, James SA, Robb E, Wikhe K et al (2010). Iron-export ferroxidase activity of beta-amyloid precursor protein is inhibited by zinc in Alzheimer's disease. Cell 142: $857-867$.

Floyd RA, Carney JM (1993). The role of metal ions in oxidative processes and aging. Toxicol Ind Health 9: 197-214.

Fratiglioni L, Launer LJ, Andersen K, Breteler MM, Copeland JR, Dartigues JF et al (2000). Incidence of dementia and major subtypes in Europe: a collaborative study of population-based cohorts. Neurology 54(11 Suppl 5): S10-S15.

Friedman A (1994). Old-onset Parkinson's disease compared with young-onset disease: clinical differences and similarities. Acta Neurol Scand 89: 258-261.

Georgieff MK (2008). The role of iron in neurodevelopment: fetal iron deficiency and the developing hippocampus. Biochem Soc Trans 36: 1267-1271. 
Good PF, Perl DP, Bierer LM, Schmeidler J (1992). Selective accumulation of aluminum and iron in the neurofibrillary tangles of Alzheimer's disease: a laser microprobe (LAMMA) study. Ann Neurol 31: 286-292.

Gossuin Y, Muller RN, Gillis P (2004). Relaxation induced by ferritin: a better understanding for an improved MRI iron quantification. NMR Biomed 17: 427-432.

Haacke EM, Cheng NY, House MJ, Liu Q, Neelavalli J, Ogg RJ et al (2005). Imaging iron stores in the brain using magnetic resonance imaging. Magn Reson Imaging 23: 1-25.

Hallgren B, Sourander P (1958). The effect of age on the nonhaemin iron in the human brain. J Neurochem 3: 41-51.

Halliwell B, Gutteridge JMC (1985). Oxygen radicals and the nervous system. Trends Neurosci 8: 22-26.

Halliwell B, Gutteridge JMC (1988). Iron as biological pro-oxidant. ISI Atlas Sci Biochem 1: 48-52.

Hider RC, Ma Y, Molina-Holgado F, Gaeta A, Roy S (2008). Iron chelation as a potential therapy for neurodegenerative disease. Biochem Soc Trans 36: 1304-1308.

House MJ, St Pierre TG, Milward EA, Bruce DG, Olynyk JK (2010). Relationship between brain $\mathrm{R}(2)$ and liver and serum iron concentrations in elderly men. Magn Reson Med 63: 275-281.

Kastman EK, Willette AA, Coe CL, Bendlin BB, Kosmatka KJ, McLaren DG et al (2010). A calorie-restricted diet decreases brain iron accumulation and preserves motor performance in old rhesus monkeys. J Neurosci 30: 7940-7947.

Kaur D, Peng J, Chinta SJ, Rajagopalan S, Di Monte DA, Cherny RA et al (2006a). Increased murine neonatal iron intake results in Parkinson-like neurodegeneration with age. Neurobiol Aging 28: 907-913.

Kaur D, Rajagopalan S, Chinta S, Kumar J, Di Monte D, Cherny RA et al (2006b). Chronic ferritin expression within murine dopaminergic midbrain neurons results in a progressive agerelated neurodegeneration. Brain Res 1140: 188-194.

Kell DB (2009). Iron behaving badly: inappropriate iron chelation as a major contributor to the aetiology of vascular and other progressive inflammatory and degenerative diseases. BMC Med Genomics 2: 2.

Klintworth GK (1973). Huntington's chorea - morphologic contributions of a century. In: Barbeau A, Paulson GW, Chase TN (eds). Advances in Neurology, Vol 1: Huntington's Chorea, 1872-1972. Raven Press: New York. pp 353-368.

Kotzbauer PT, Trojanowsk JQ, Lee VM (2001). Lewy body pathology in Alzheimer's disease. J Mol Neurosci 17: 225-232.

Kraemer HC, Yesavage JA, Taylor JL, Kupfer D (2000). How can we learn about developmental processes from cross-sectional studies, or can we? Am J Psychiatry 157: 163-171.

Landau SM, Lal R, O’Neil JP, Baker S, Jagust WJ (2009). Striatal dopamine and working memory. Cereb Cortex 19: 445-454.

Lavados M, Guillon M, Mujica MC, Rojo LE, Fuentes P, Maccioni RB (2008). Mild cognitive impairment and Alzheimer patients display different levels of redox-active CSF iron. J Alzheimers Dis 13: $225-232$

Lee SY, Patton SM, Henderson RJ, Connor JR (2007). Consequences of expressing mutants of the hemochromatosis gene (HFE) into a human neuronal cell line lacking endogenous HFE. FASEB J 21: 564-576.

Lehmann DJ, Schuur M, Warden DR, Hammond N, Belbin O, Kolsch $\mathrm{H}$ et al (2010). Transferrin and HFE genes interact in Alzheimer's disease risk: the Epistasis Project. Neurobiol Aging. doi:10.1016/j.neurobiolaging.2010.07.018.

Lewis SJ, Dove A, Robbins TW, Barker RA, Owen AM (2004). Striatal contributions to working memory: a functional magnetic resonance imaging study in humans. Eur J Neurosci 19: 755-760.

Li L, Fang CJ, Ryan JC, Niemi EC, Lebron JA, Bjorkman PJ et al (2010). Binding and uptake of $\mathrm{H}$-ferritin are mediated by human transferrin receptor-1. Proc Natl Acad Sci USA 107: $3505-3510$.
Lobo A, Launer LJ, Fratiglioni L, Andersen K, Di Carlo A, Breteler MM et al (2000). Prevalence of dementia and major subtypes in Europe: a collaborative study of population-based cohorts. Neurology 54(11 Suppl 5): S4-S9.

Ma L, Wang W, Zhao M, Li M (2008). Foot-shock stress-induced regional iron accumulation and altered iron homeostatic mechanisms in rat brain. Biol Trace Elem Res 126: 204-213.

Maaroufi K, Had-Aissouni L, Melon C, Sakly M, Abdelmelek H, Poucet B et al (2009). Effects of prolonged iron overload and low frequency electromagnetic exposure on spatial learning and memory in the young rat. Neurobiol Learn Mem 92: 345-355.

Mandel SA, Amit T, Kalfon L, Reznichenko L, Weinreb O, Youdim MB (2008). Cell signaling pathways and iron chelation in the neurorestorative activity of green tea polyphenols: special reference to epigallocatechin gallate (EGCG). J Alzheimers Dis 15: $211-222$.

Massie HR, Aiello VR, Williams TR (1985). Iron accumulation during development and ageing of Drosophila. Mech Ageing Dev 29: 215-220.

Massie HR, Aiello VR, Williams TR (1993). Inhibition of iron absorption prolongs the life span of Drosophila. Mech Ageing Dev 67: 227-237.

Miech RA, Breitner JC, Zandi PP, Khachaturian AS, Anthony JC, Mayer L (2002). Incidence of AD may decline in the early 90s for men, later for women: the Cache County Study. Neurology 58: 209-218.

Mitchell RM, Lee SY, Simmons Z, Connor JR (2009). HFE polymorphisms affect cellular glutamate regulation. Neurobiol Aging doi:10.1016/j.neurobiolaging.2009.05.016.

Moalem S, Percy ME, Andrews DF, Kruck TP, Wong S, Dalton AJ et al (2000). Are hereditary hemochromatosis mutations involved in Alzheimer disease? AM J Med Genet 93: 58-66.

Morris CM, Candy JM, Oakley AE, Bloxham CA, Edwardson JA (1992). Histochemical distribution of non-haem iron in the human brain. Acta Anat (Basel) 144: 235-257.

Njajou OT, Alizadeh BZ, Aulchenko Y, Zillikens MC, Pols HA, Oostra BA et al (2006). Heritability of serum iron, ferritin and transferrin saturation in a genetically isolated population, the Erasmus Rucphen Family (ERF) Study. Hum Hered 61: 222-228.

Pankhurst Q, Hautot D, Khan N, Dobson J (2008). Increased levels of magnetic iron compounds in Alzheimer's disease. J Alzheimers Dis 13: 49-52.

Pantelatos A, Fornadi F (1993). Clinical features and medical treatment of Parkinson's disease in patient groups selected in accordance with age at onset. Adv Neurol 60: 690-697.

Peterson LR, Peterson MJ (1959). Short-term retention of individual verbal items. J Exp Psychol 58: 193-198.

Pfefferbaum A, Adalsteinsson E, Rohlfing T, Sullivan EV (2009). MRI estimates of brain iron concentration in normal aging: comparison of field-dependent (FDRI) and phase (SWI) methods. Neuroimage 47: 493-500.

Raber J, Huang Y, Ashford JW (2004). ApoE genotype accounts for the vast majority of AD risk and AD pathology. Neurobiol Aging 25: 641-650.

Rajendran R, Minqin R, Ynsa MD, Casadesus G, Smith MA, Perry $\mathrm{G}$ et al (2009). A novel approach to the identification and quantitative elemental analysis of amyloid deposits-insights into the pathology of Alzheimer's disease. Biochem Biophys Res Commun 382: 91-95.

Reitan R, Wolfson D (1985). The Halstead-Reitan Neuropsychological Test Battery. Neuropsychology Press: Tucson, AZ.

Roth JA, Singleton S, Feng J, Garrick M, Paradkar PN (2010). Parkin regulates metal transport via proteasomal degradation of the 1B isoforms of divalent metal transporter 1 (DMT1). J Neurochem 113: 454-464.

Sampietro M, Caputo L, Casatta A, Meregalli M, Pellagatti A, Tagliabue J et al (2001). The hemochromatosis gene affects the 
age of onset of sporadic Alzheimer's disease. Neurobiol Aging 22: 563-568.

Schmidt AT, Waldow KJ, Grove WM, Salinas JA, Georgieff MK (2007). Dissociating the long-term effects of fetal/neonatal iron deficiency on three types of learning in the rat. Behav Neurosci 121: 475-482.

Shoham S, Youdim MB (2002). The effects of iron deficiency and iron and zinc supplementation on rat hippocampus ferritin. J Neural Transm 109: 1241-1256.

Siddappa AM, Georgieff MK, Wewerka S, Worwa C, Nelson CA, Deregnier RA (2004). Iron deficiency alters auditory recognition memory in newborn infants of diabetic mothers. Pediatr Res 55: 1034-1041.

Simpson EH, Kellendonk C, Kandel E (2010). A possible role for the striatum in the pathogenesis of the cognitive symptoms of schizophrenia. Neuron 65: 585-596.

Singh A, Isaac AO, Luo X, Mohan ML, Cohen ML, Chen F et al (2009). Abnormal brain iron homeostasis in human and animal prion disorders. PLoS Pathog 5: e1000336.

Smith MA, Harris PL, Sayre LM, Perry G (1997). Iron accumulation in Alzheimer disease is a source of redox-generated free radicals. Proc Natl Acad Sci USA 94: 9866-9868.

Smith MA, Zhu X, Tabaton M, Liu G, McKeel Jr DW, Cohen ML et al (2010). Increased iron and free radical generation in preclinical Alzheimer disease and mild cognitive impairment. J Alzheimers Dis 19: 363-372.

Squire LR, Zola-Morgan S (1991). The medial temporal lobe memory system. Science 253: 1380-1386.

Sullivan JL (1989). The iron paradigm of ischemic heart disease [see comments]. Am Heart J 117: 1177-1188.

Thompson CM, Markesbery WR, Ehmann WD, Mao YX, Vance DE (1988). Regional brain trace-element studies in Alzheimer's disease. Neurotoxicology 9: 1-7.

Valle A, Silvestri E, Moreno M, Chambery A, Oliver J, Roca P et al (2008). Combined effect of gender and caloric restriction on liver proteomic expression profile. J Proteome Res 7: 2872-2881.

Vinet J, Lemieux P, Tamburri A, Tiesinga P, Scafidi J, Gallo V et al (2010). Subclasses of oligodendrocytes populate the mouse hippocampus. Eur J Neurosci 31: 425-438.
Vymazal J, Brooks RA, Baumgarner C, Tran V, Katz D, Bulte JW et al (1996a). The relation between brain iron and NMR relaxation times: an in vitro study. Magn Reson Med 35: 56-61.

Vymazal J, Brooks RA, Patronas N, Hajek M, Bulte JW, Di Chiro G (1995a). Magnetic resonance imaging of brain iron in health and disease. J Neurol Sci 134(Suppl): 19-26.

Vymazal J, Hajek M, Patronas N, Giedd JN, Bulte JW, Baumgarner $\mathrm{C}$ et al (1995b). The quantitative relation between T1-weighted and T2-weighted MRI of normal gray matter and iron concentration. J Magn Reson Imaging 5: 554-560.

Vymazal J, Zak O, Bulte JW, Aisen P, Brooks RA (1996b). T1 and T2 of ferritin solutions: effect of loading factor. Magn Reson Med 36: 61-65.

Wechsler D (1981). Wechsler Adult Intelligence Scale-Revised. Psychological Corporation: San Antonio.

Wechsler D (1997). Wechsler Adult Intelligence Scale-III: Manual. 3rd edn. Psychological Corporation: San Antonio.

Whitfield JB, Cullen LM, Jazwinska EC, Powell LW, Heath AC, Zhu $\mathrm{G}$ et al (2000). Effects of HFE C282Y and H63D polymorphisms and polygenic background on iron stores in a large community sample of twins. Am J Hum Genet 66: 1246-1258.

Whitfield JB, Treloar S, Zhu G, Powell LW, Martin NG (2003). Relative importance of female-specific and non-female-specific effects on variation in iron stores between women. Br J Haematol 120: $860-866$.

Xu J, Knutson MD, Carter CS, Leeuwenburgh C (2008). Iron accumulation with age, oxidative stress and functional decline. PLoS One 3: e2865.

Yao B, Li TQ, Gelderen P, Shmueli K, de Zwart JA, Duyn JH (2009). Susceptibility contrast in high field MRI of human brain as a function of tissue iron content. Neuroimage 44: 1259-1266.

Zecca L, Casella L, Albertini A, Bellei C, Zucca FA, Engelen M et al (2008). Neuromelanin can protect against iron mediated oxidative damage in system modeling iron overload of brain aging and Parkinson's disease. J Neurochem 106: 1866-1875.

Zecca L, Youdim MB, Riederer P, Connor JR, Crichton RR (2004). Iron, brain ageing and neurodegenerative disorders. Nat Rev Neurosci 5: 863-873. 